\title{
Analytic Solution of Steady Three-Dimensional Problem of Condensation Film on Inclined Rotating Disk by Differential Transform Method
}

\author{
Mohammad Mehdi Rashidi and Seyyed Amin Mohimanian pour
}

Engineering Faculty of Bu-Ali Sina University, P.O. Box 65175-4161, Hamedan, Iran

Correspondence should be addressed to Mohammad Mehdi Rashidi,mm_rashidi@yahoo.com

Received 2 February 2010; Accepted 3 August 2010

Academic Editor: Dane Quinn

Copyright (C) 2010 M. M. Rashidi and S. A. Mohimanian pour. This is an open access article distributed under the Creative Commons Attribution License, which permits unrestricted use, distribution, and reproduction in any medium, provided the original work is properly cited.

The differential transform method (DTM) is applied to the steady three-dimensional problem of a condensation film on an inclined rotating disk. With similarity method, the governing equations can be reduced to a system of nonlinear ordinary differential equations. The approximate solutions of these equations are obtained in the form of series with easily computable terms. The velocity and temperature profiles are shown and the influence of Prandtl number on the temperature profiles is discussed in detail. The validity of our solutions is verified by the numerical results.

\section{Introduction}

Most phenomena in our world are essentially nonlinear and are described by nonlinear equations. Some of them are solved using numerical methods and some are solved using the analytic methods of perturbation [1,2]. The numerical methods give discontinuous points of a curve and thus it is often costly and time consuming to get a complete curve of results and so in these methods, stability and convergence should be considered so as to avoid divergence or inappropriate results. Besides, from numerical results, it is hard to have a whole and essential understanding of a nonlinear problem. Numerical difficulties additionally appear if a nonlinear problem contains singularities or has multiple solutions. In the analytic perturbation methods, we should exert the small parameter in the equation. Therefore, finding the small parameter and exerting it into the equation are deficiencies of the perturbation methods. Recently, much attention has been devoted to the newly developed methods to construct approximate analytic solutions of nonlinear equations without mentioned deficiencies. 
One of the semiexact methods which does not need small parameters is the DTM; this method was first proposed by Zhou [3], who solved linear and nonlinear problems in electrical circuit problems. Chen and Ho [4] developed this method for partial differential equations and Ayaz [5] applied it to the system of differential equations; this method is very powerful [6]. This method constructs an analytical solution in the form of a polynomial. It is different from the traditional higher-order Taylor series method. The Taylor series method is computationally expensive for large orders. The DTM is an alternative procedure for obtaining analytic Taylor series solution of the differential equations. In recent years, the DTM has been successfully employed to solve many types of nonlinear problems [7-14].

Removing of a condensate liquid from a cooled, saturated vapor is important in engineering processes. Sparrow and Gregg [15] considered the removal of the condensate using centrifugal forces on a cooled rotating disk. Followingvon Karman's [16] study of a rotating disk in an infinite fluid, Sparrow and Gregg transformed the Navier-Stokes equations into a set of nonlinear ordinary differential equations and numerically integrated for the similarity solution for several finite film thicknesses. Their work was extended by adding vapor drag by Beckett et al. [17] and adding suction on the plate by Chary and Sarma [18]. The problem is also related to chemical vapor deposition, when a thin fluid film is deposited on a cooled rotating disk [19].

In this paper, the DTM is applied to find the totally analytic solution for the problem of condensation or spraying on an inclined rotating disk and is compared with the numerical solution and the homotopy analysis method (HAM). This problem was studied first by Wang [20] in 2006, andRashidi and Dinarvand [21] applied the HAM for it; please also see [22, 23]. In this way, the paper has been organized as follows: in Section 2, the flow analysis and mathematical formulation are presented. In Section 3, we extend the application of the DTM to construct the approximate solutions for the governing equations. Section 4 contains the results and discussion. The conclusions are summarized in Section 5.

\section{Flow Analysis and Mathematical Formulation}

Figure 1 shows a disk rotating in its own plane with angular velocity $\Omega$. The angle between horizontal axis and disk is $\beta$. A fluid film of thickness $h$ is formed by spraying, with the $W$ velocity. We assume the disk radius is large compared to the film thickness such that the end effects can be ignored. Vapor shear effects at the interface of vapor and fluid are usually also unimportant. The gravitational acceleration, $\bar{g}$, acts in the downward direction. The temperature on the disk is $T_{w}$ and the temperature on the film surface is $T_{0}$. Besides, the ambient pressure on the film surface is constant at $p_{0}$ and we can safely say the pressure is a function of $z$ only. Neglecting viscous dissipation, the continuity, momentum and energy equations for steady state are given in the following form:

$$
\begin{gathered}
u_{x}+v_{y}+w_{z}=0 \\
u u_{x}+v u_{y}+w u_{z}=v\left(u_{x x}+u_{y y}+u_{z z}\right)+\bar{g} \sin \beta, \\
u v_{x}+v v_{y}+w v_{z}=v\left(v_{x x}+v_{y y}+v_{z z}\right), \\
u w_{x}+v w_{y}+w w_{z}=v\left(w_{x x}+w_{y y}+w_{z z}\right)-\bar{g} \cos \beta-\frac{p_{z}}{\rho}, \\
u T_{x}+v T_{y}+w T_{z}=\alpha\left(T_{x x}+T_{y y}+T_{z z}\right) .
\end{gathered}
$$




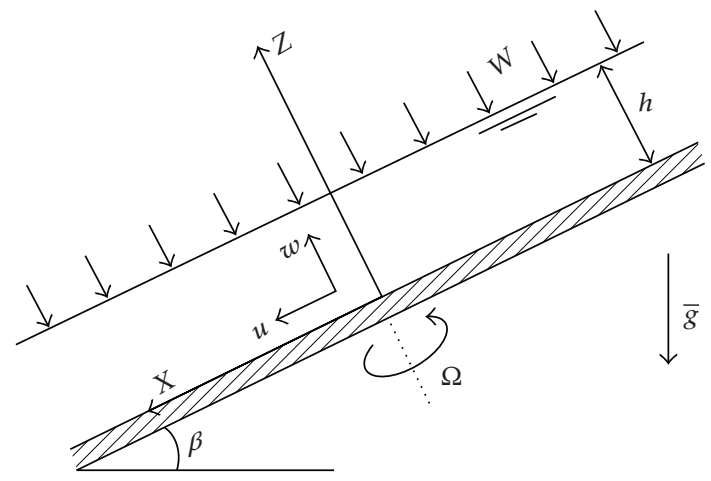

Figure 1: Schematic diagram of the problem.

In above equations, $u, v$, and $w$ indicate the velocity components in the $x, y$, and $z$ directions, respectively; $T$ denotes the temperature, $\rho, v$, and $\alpha$ are the density, kinematic viscosity and thermal diffusivity of the fluid, respectively. Supposing zero slip on the disk and zero shear stress on the film surface, the boundary conditions are

$$
\begin{gathered}
u=-\Omega y, \quad v=\Omega x, \quad w=0, \quad T=T_{w} \text { at } z=0, \\
u_{z}=0, \quad v_{z}=0, \quad w=-W, \quad T=T_{0}, \quad p=p_{0} \text { at } z=h .
\end{gathered}
$$

For the mentioned problem, Wang introduced the following transform [20]:

$$
\begin{aligned}
& u=-\Omega y g(\eta)+\Omega x f^{\prime}(\eta)+\bar{g} k(\eta) \sin \frac{\beta}{\Omega^{\prime}} \\
& v=\Omega x g(\eta)+\Omega y f^{\prime}(\eta)+\bar{g} s(\eta) \sin \frac{\beta}{\Omega^{\prime}} \\
& w=-2 \sqrt{\Omega v} f(\eta), \\
& T=\left(T_{0}-T_{w}\right) \theta(\eta)+T_{w},
\end{aligned}
$$

where

$$
\eta=z \sqrt{\frac{\Omega}{v}} .
$$

Continuity (2.1) automatically is satisfied. Equations (2.2) and (2.3) can be written as follows:

$$
\begin{gathered}
f^{\prime \prime \prime}-\left(f^{\prime}\right)^{2}+g^{2}+2 f f^{\prime \prime}=0, \\
g^{\prime \prime}-2 g f^{\prime}+2 f g^{\prime}=0, \\
k^{\prime \prime}-k f^{\prime}+s g+2 f k^{\prime}+1=0, \\
s^{\prime \prime}-g k-s f^{\prime}+2 f s^{\prime}=0,
\end{gathered}
$$


If the temperature is a function of the distance $z$ only, (2.5) becomes

$$
\theta^{\prime \prime}+2 \operatorname{Pr} f \theta^{\prime}=0
$$

where $\operatorname{Pr}=v / \alpha$ is the Prandtl number. The boundary conditions for (2.9)-(2.13) are

$$
\begin{array}{ll}
f(0)=0, & f^{\prime}(0)=0, \quad f^{\prime \prime}(\delta)=0, \\
g(0)=1, & g^{\prime}(\delta)=0, \\
k(0)=0, & k^{\prime}(\delta)=0 \\
s(0)=0, & s^{\prime}(\delta)=0 \\
\theta(0)=0, & \theta(\delta)=1,
\end{array}
$$

and $\delta$ is the constant normalized thickness as

$$
\delta=h \sqrt{\Omega / v}
$$

After the flow field is found, the pressure can be obtained by integrating (2.4)

$$
p(z)=p_{0}+\rho\left\{v\left[w_{z}(z)-w_{z}(h)\right]-\left[w^{2}(z)-w^{2}(h)\right] / 2-\bar{g}(z-h) \cos \beta\right\} .
$$

\section{The Differential Transform Method}

Basic definitions and operations of differential transformation are introduced as follows. Differential transformation of the function $f(\eta)$ is defined as follows:

$$
F(k)=\frac{1}{k !}\left[\frac{d^{k} f(\eta)}{d \eta^{k}}\right]_{\eta=\eta_{0}},
$$

in (3.1), $f(\eta)$ is the original function and $F(k)$ is the transformed function which is called the T-function (it is also called the spectrum of the $f(\eta)$ at $\eta=\eta_{0}$, in the $K$ domain). The differential inverse transformation of $F(k)$ is defined as

$$
f(\eta)=\sum_{k=0}^{\infty} F(k)\left(\eta-\eta_{0}\right)^{k}
$$

combining (3.1) and (3.2), we obtain

$$
f(\eta)=\sum_{k=0}^{\infty}\left[\frac{d^{k} f(\eta)}{d \eta^{k}}\right]_{\eta=\eta_{0}} \frac{\left(\eta-\eta_{0}\right)^{k}}{k !}
$$

equation (3.3) implies that the concept of the differential transformation is derived from Taylor's series expansion, but the method does not evaluate the derivatives symbolically. 
However, relative derivatives are calculated by an iterative procedure that is described by the transformed equations of the original functions.

From the definitions of (3.1) and (3.2), it is easily proven that the transformed functions comply with the basic mathematical operations shown in below. In real applications, the function $f(\eta)$ in (3.2) is expressed by a finite series and can be written as

$$
f(\eta)=\sum_{k=0}^{N} F(k)\left(\eta-\eta_{0}\right)^{k}
$$

equation (3.4) implies that $\sum_{k=N+1}^{\infty} F(k)\left(\eta-\eta_{0}\right)^{k}$ is negligibly small, where $N$ is series size.

Theorems to be used in the transformation procedure, which can be evaluated from (3.1) and (3.2), are given below.

Theorem 3.1. If $f(\eta)=g(\eta) \pm h(\eta)$, then $F(k)=G(k) \pm H(k)$.

Theorem 3.2. If $f(\eta)=c g(\eta)$, then $F(k)=c G(k)$, where $c$ is a constant.

Theorem 3.3. If $f(\eta)=d^{n} g(\eta) / d \eta^{n}$, then $F(k)=((k+n) ! / k !) G(k+n)$.

Theorem 3.4. If $f(\eta)=g(\eta) h(\eta)$, then $F(k)=\sum_{l=0}^{k} G(l) H(k-l)$.

Theorem 3.5. If $f(\eta)=\eta^{n}$,

$$
F(k)=\delta_{D}(k-n), \quad \text { where } \delta_{D}(k-n)= \begin{cases}1, & k=n \\ 0, & k \neq 0\end{cases}
$$

Taking differential transform of (2.9)-(2.13), the following equations can be obtained

$$
\begin{aligned}
& (k+1)(k+2)(k+3) F(k+3)-\sum_{r=0}^{k}[(k+1-r)(r+1) F(r+1) F(k+1-r)] \\
& +\sum_{r=0}^{k}[G(r) G(k-r)]+2 \sum_{r=0}^{k}[(k+2-r)(k+1-r) F(k+2-r) F(r)]=0, \\
& (k+2)(k+1) G(k+2)-2 \sum_{r=0}^{k}[(k+1-r) G(r) F(k+1-r)] \\
& \quad+2 \sum_{r=0}^{k}(k+1-r) F(r) G(k+1-r)=0, \\
& (k+2)(k+1) K(k+2)-2 \sum_{r=0}^{k}[(k+1-r) K(r) F(k+1-r)]+\sum_{r=0}^{k}[S(r) G(k-r)] \\
& \quad+2 \sum_{r=0}^{k}[(k+1-r) F(r) K(k+1-r)]+\delta_{D}(k)=0,
\end{aligned}
$$




$$
\begin{aligned}
& (k+2)(k+1) S(k+2)-\sum_{r=0}^{k}[G(r) K(k-r)]-\sum_{r=0}^{k}[(k+1-r) S(r) F(k+1-r)] \\
& \quad+2 \sum_{r=0}^{k}(k+1-r) F(r) S(k+1-r)=0, \\
& (k+2)(k+1) \Theta(k+2)+2 \operatorname{Pr} \sum_{r=0}^{k}[(k+1-r) F(r) \Theta(k+1-r)]=0,
\end{aligned}
$$

where $F(k), G(k), K(k), S(k)$ and $\Theta(k)$ are the differential transforms of $f(\eta), g(\eta), k(\eta)$, $s(\eta)$ and $\theta(\eta)$. The transforms of the boundary conditions are

$$
\begin{array}{rlrl}
F(0) & =0, & F(1)=0, & F(2)=\gamma_{1}, \\
G(0)=1, & G(1)=\gamma_{2}, & \\
K(0)=0, & K(1)=\gamma_{3}, \\
S(0)=0, & S(1)=\gamma_{4}, \\
\Theta(0)=0, & \Theta(1)=\gamma_{5},
\end{array}
$$

where $\gamma_{i}(i=1, \ldots, 5)$ are constants. For these constants, the problem is solved with (3.7) and then the boundary conditions (2.14) are applied

$$
f^{\prime \prime}(\delta)=0, \quad \mathrm{~g}(\delta)=0, \quad k(\delta)=0, \quad s(\delta)=0, \quad \theta(\delta)=1 .
$$

For $\delta=0.5, N=20$, and $\operatorname{Pr}=7$, we have

$$
\begin{gathered}
\gamma_{1}=0.24126840, \quad \gamma_{2}=-0.07866202, \quad \gamma_{3}=-0.04009537, \\
\gamma_{4}=-0.49411375, \quad \gamma_{5}=2.05582108,
\end{gathered}
$$

and the solutions of above equations (using the DTM) are as follows:

$$
\begin{aligned}
f(\eta) \approx & 0.241268 \eta^{2}-0.16666667 \eta^{3}+0.00655517 \eta^{4}-0.000103129 \eta^{5}-0.00134038 \eta^{6} \\
& +0.000457075 \eta^{7}-0.0000309189 \eta^{8}-0.0000146493 \eta^{9}+0.0000132522 \eta^{10} \\
& -2.92946 \times 10^{-6} \eta^{11}+1.01173 \times 10^{-6} \eta^{12}-9.04629 \times 10^{-7} \eta^{13}+3.76003 \times 10^{-7} \eta^{14} \\
& -9.76475 \times 10^{-8} \eta^{15}+4.35162 \times 10^{-8} \eta^{16}-2.3391 \times 10^{-8} \eta^{17}+8.10052 \times 10^{-9} \eta^{18} \\
& -2.49821 \times 10^{-9} \eta^{19}+1.08501 \times 10^{-9} \eta^{20}
\end{aligned}
$$




$$
\begin{aligned}
& g(\eta) \approx 1-0.078662 \eta+0.160846 \eta^{3}-0.0864964 \eta^{4}+0.00524414 \eta^{5}-0.00272464 \eta^{6} \\
& +0.00160609 \eta^{7}-0.000479669 \eta^{8}+0.000107813 \eta^{9}-0.0000908193 \eta^{10} \\
& +0.000044882 \eta^{11}-0.0000122088 \eta^{12}+3.99763 \times 10^{-6} \eta^{13}-2.17641 \times 10^{-6} \eta^{14} \\
& +8.28203 \times 10^{-7} \eta^{15}-2.3808 \times 10^{-7} \eta^{16}+8.39076 \times 10^{-8} \eta^{17}-3.48713 \times 10^{-8} \eta^{18} \\
& +1.16737 \times 10^{-8} \eta^{19}-3.36121 \times 10^{-9} \eta^{20}, \\
& k(\eta) \approx 0.494114 \eta-0.5 \eta^{2}+0.00668256 \eta^{3}-0.000262832 \eta^{4}+0.00382819 \eta^{5} \\
& -0.000849056 \eta^{6}-0.000144438 \eta^{7}-0.000412106 \eta^{8}+0.000326912 \eta^{9} \\
& -0.0000725119 \eta^{10}+0.0000170146 \eta^{11}-0.0000173481 \eta^{12}+7.70124 \times 10^{-6} \eta^{13} \\
& -1.83103 \times 10^{-6} \eta^{14}+7.05439 \times 10^{-7} \eta^{15}-1.23679 \times 10^{-6} \eta^{16}+4.90306 \times 10^{-7} \eta^{17} \\
& -1.93933 \times 10^{-7} \eta^{18}+8.19309 \times 10^{-8} \eta^{19}-3.38424 \times 10^{-8} \eta^{20}, \\
& s(\eta) \approx-0.0400954 \eta+0.0823523 \eta^{3}-0.0449057 \eta^{4}+0.00263481 \eta^{5}-0.0000438053 \eta^{6} \\
& -0.000312347 \eta^{7}+0.0000427222 \eta^{8}-5.44922 \times 10^{-6} \eta^{9}+0.0000350801 \eta^{10} \\
& -0.0000178174 \eta^{11}+3.30275 \times 10^{-6} \eta^{12}-2.08021 \times 10^{-6} \eta^{13}+1.83037 \times 10^{-6} \eta^{14} \\
& -6.80866 \times 10^{-7} \eta^{15}+1.99374 \times 10^{-7} \eta^{16}-1.10268 \times 10^{-7} \eta^{17}+5.62782 \times 10^{-8} \eta^{18} \\
& -1.9123 \times 10^{-8} \eta^{19}+6.79324 \times 10^{-9} \eta^{20}, \\
& \theta(\eta) \approx 2.05582 \eta-0.578672 \eta^{4}+0.239846 \eta^{5}-0.00628892 \eta^{6}+0.186224 \eta^{7}-0.16809 \eta^{8} \\
& +0.0434017 \eta^{9}-0.0511522 \eta^{10}+0.0686 \eta^{11}-0.0343928 \eta^{12}+0.0176617 \eta^{13} \\
& -0.0206345 \eta^{14}+0.0150924 \eta^{15}-0.00758758 \eta^{16}+0.0057454 \eta^{17}-0.00479229 \eta^{18} \\
& +0.00281121 \eta^{19}-0.00165154 \eta^{20} \text {. }
\end{aligned}
$$

\section{Results and Discussion}

Graphical representation of the obtained results is very useful to demonstrate the efficiency and accuracy of the DTM for above problem. Figures 2-7 show the normalized velocity profiles $f(\eta), f^{\prime}(\eta), g(\eta), k(\eta)$, and $s(\eta)$ obtained by the DTM and the HAM (5th-order approximation) in comparison with the numerical solution by the fifth-order Runge-Kutta method. The HAM solutions are obtained from [21]. In Figures 2-7, we can see a very good agreement between the DTM and numerical results. In Table 1, the analytical results of DTM and HAM are compared with numerical method. Table 1 indicates that the results obtained by the DTM have six digits precision with the numerical solutions. So, it is obvious that the DTM reaches a very high accuracy in comparison with the HAM for similar values of series size (On the other hand, the accuracy of the HAM results increases when the order of approximation increases). In Figure 8, CPU time is considered for two methods. This figure 


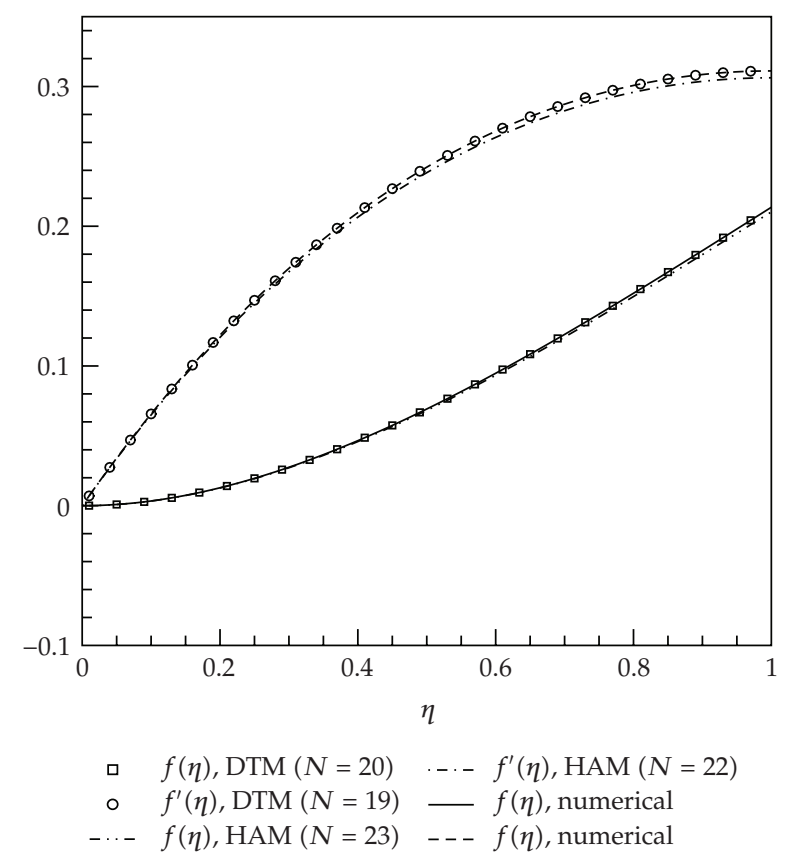

Figure 2: The normalized radial velocity profiles $\left(f(\eta)\right.$ and $f^{\prime}(\eta)$ ) for the rotating flow obtained by the DTM and the HAM (5th-order approximation) in comparison with the numerical solution, when $\delta=1$.

elucidates that CPU time of the DTM is smaller than the HAM. It should be mentioned that the DTM has some problems in solving the boundary value problems which have boundary conditions at infinity; on the other hand, with the HAM one can easily solve this kind of problems.

The normalized temperature profiles for different values of the Prandtl number are represented in Figure 9. The Prandtl number ranges from 0.01 for liquid metals, to 7 for water and to more than 100 for some oils. From Figure 9, it can be seen that the DTM solution contains all of groups. This figure elucidates that series size increase with Pandtl number. In Figure 10, the DTM solutions of the normalized temperature profiles are obtained for different values of series size.

\section{Conclusions}

In this paper, the DTM was applied successfully to find the analytical solution of steady three-dimensional problem of condensation film on inclined rotating disk. The results show that the differential transform method does not require small parameters in the equations, so the limitations of the traditional perturbation methods can be eliminated. The reliability of the method and reduction in the size of computational domain give this method a wider applicability. Therefore, this method can be applied to many nonlinear integral and differential equations without linearization, discretization or perturbation. It should be mentioned that the DTM has some problems in solving the boundary value problems which have boundary conditions at infinity, on the other hand with the HAM one can easily solve this kind of problems. 


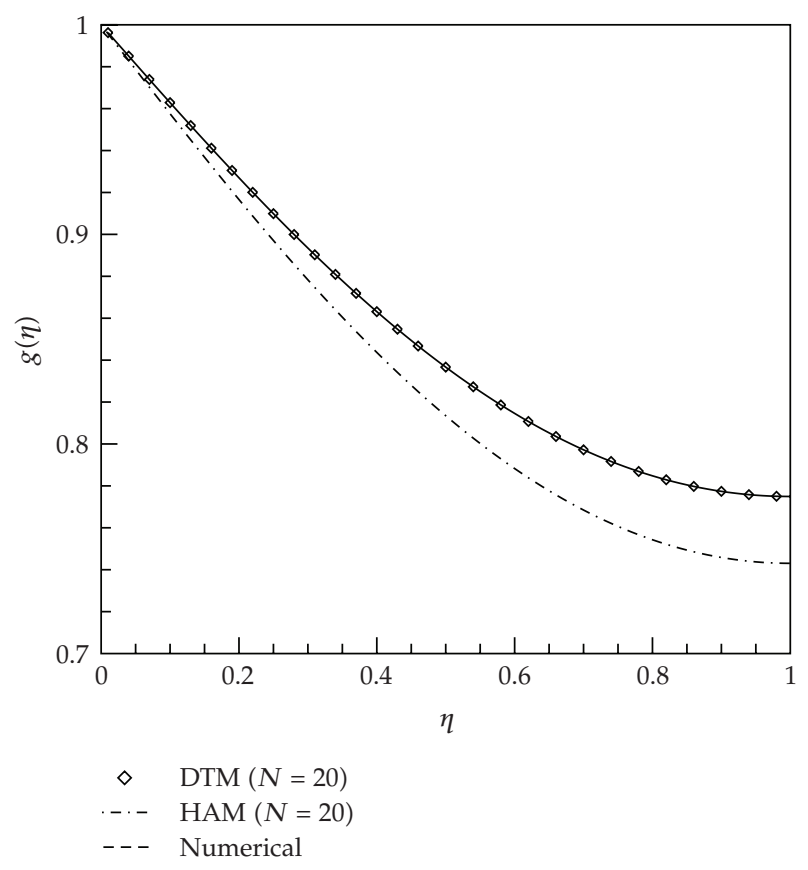

Figure 3: The normalized radial velocity profiles $(g(\eta))$ for the rotating flow obtained by the DTM and the HAM (5th-order approximation) in comparison with the numerical solution, when $\delta=1$.

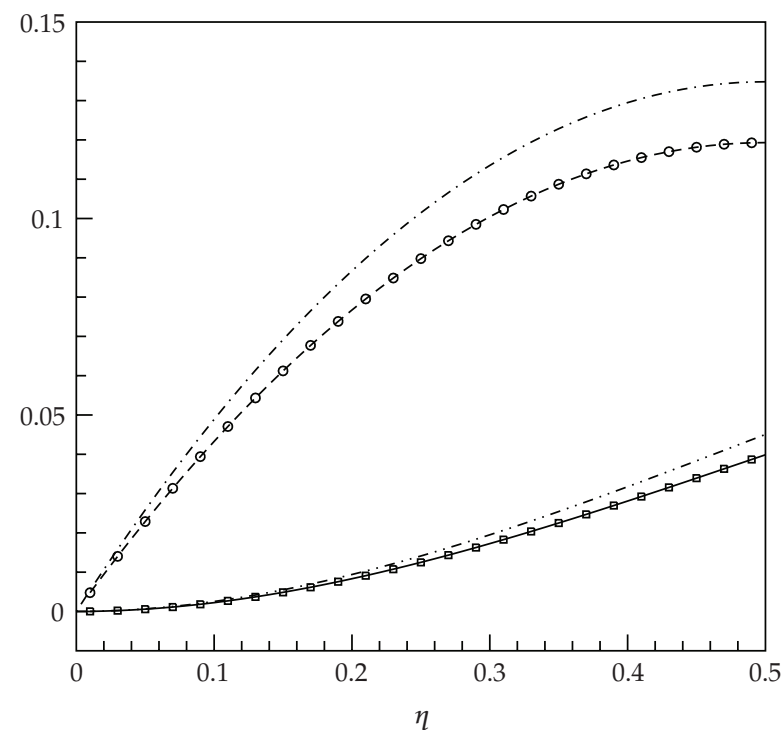
ㅁ $f(\eta), \operatorname{DTM}(N=20)$
$\cdot \cdot-f^{\prime}(\eta), \operatorname{HAM}(N=22)$
- $f^{\prime}(\eta), \operatorname{DTM}(N=19)$
$f(\eta)$, numerical
$-\cdots-f(\eta), \operatorname{HAM}(N=23)$
- - $f(\eta)$, numerical

Figure 4: The normalized radial velocity profiles $\left(f(\eta)\right.$ and $\left.f^{\prime}(\eta)\right)$ for the rotating flow obtained by the DTM and the HAM (5th-order approximation) in comparison with the numerical solution, when $\delta=0.5$. 


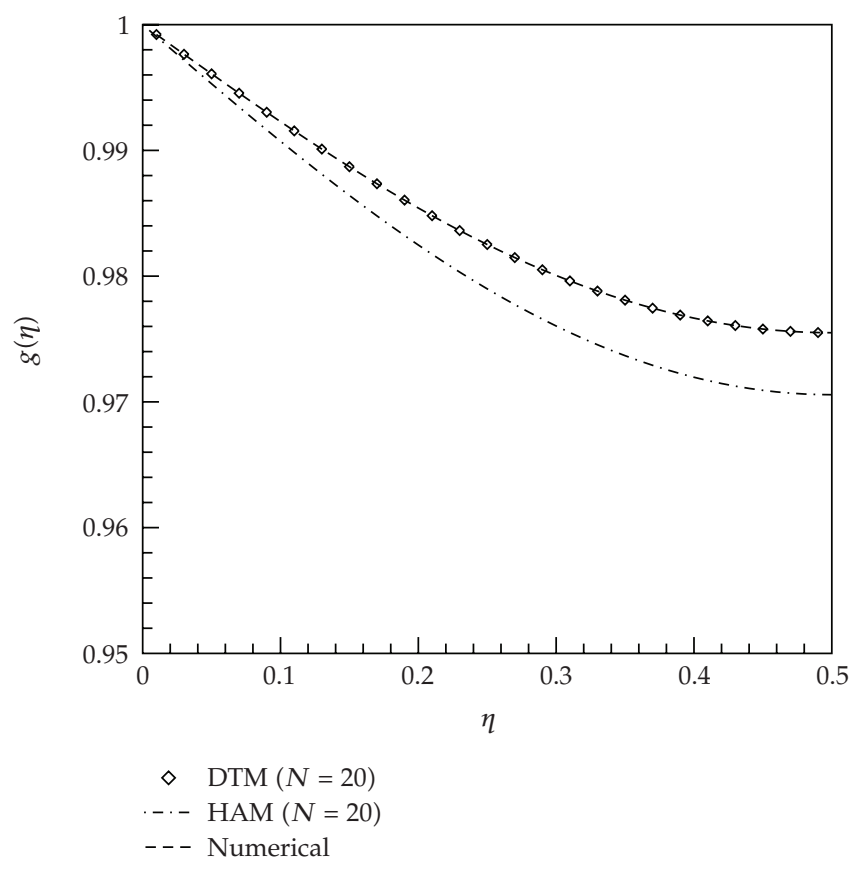

Figure 5: The normalized radial velocity profiles $(g(\eta))$ for the rotating flow obtained by the DTM and the HAM (5th-order approximation) in comparison with the numerical solution, when $\delta=0.5$.

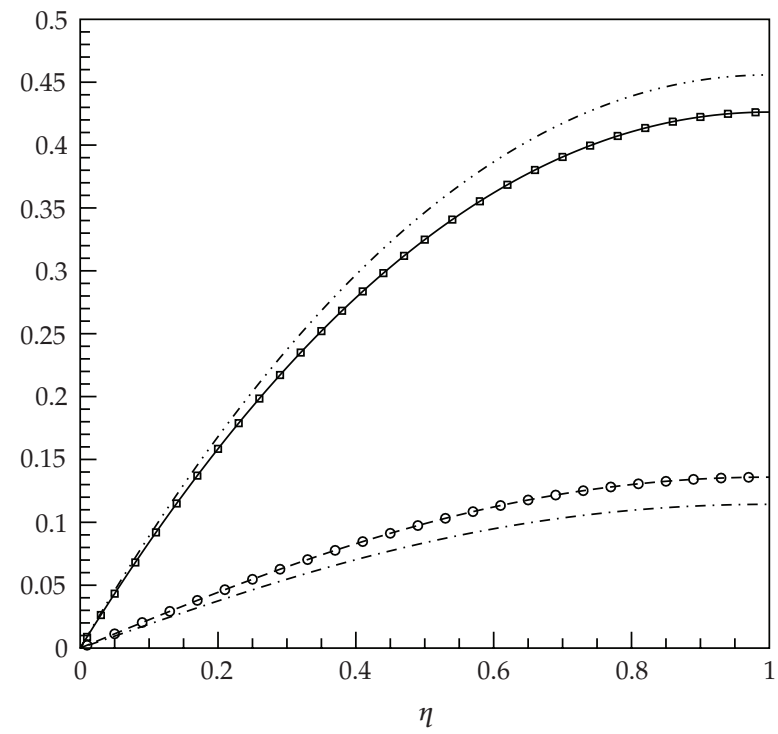

$$
\begin{array}{cccc}
\square & k(\eta), \operatorname{DTM}(N=20) & \cdots & -s(\eta), \operatorname{HAM}(N=22) \\
\circ & -s(\eta), \operatorname{DTM}(N=20) & - & k(\eta), \text { numerical } \\
\cdots & k(\eta), \operatorname{HAM}(N=22) & --- & -s(\eta), \text { numerical }
\end{array}
$$

Figure 6: The normalized velocity profiles for the draining flow $(k(\eta))$ and lateral flow $(s(\eta))$ obtained by the DTM and the HAM (5th-order approximation) in comparison with the numerical solution, when $\delta=1$. 


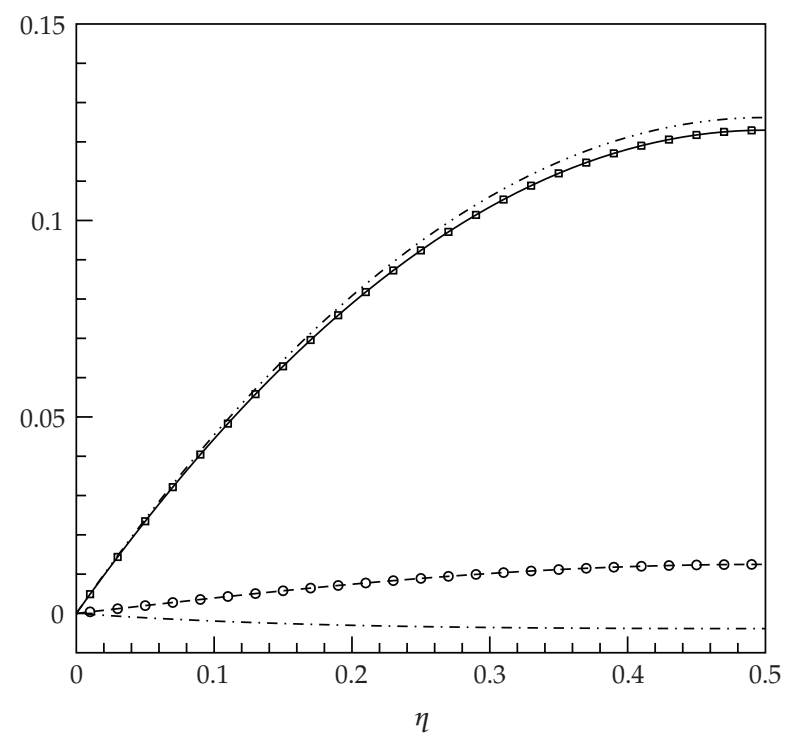

$$
\begin{array}{cccc}
\square & k(\eta), \operatorname{DTM}(N=20) & \cdots & -s(\eta), \operatorname{HAM}(N=22) \\
\circ & -s(\eta), \operatorname{DTM}(N=20) & - & k(\eta), \text { numerical } \\
\cdots & k(\eta), \operatorname{HAM}(N=22) & --- & -s(\eta), \text { numerical }
\end{array}
$$

Figure 7: The normalized velocity profiles for the draining flow $(k(\eta))$ and lateral flow $(s(\eta))$ obtained by the DTM and the HAM (5th-order approximation) in comparison with the numerical solution, when $\delta=0.5$.

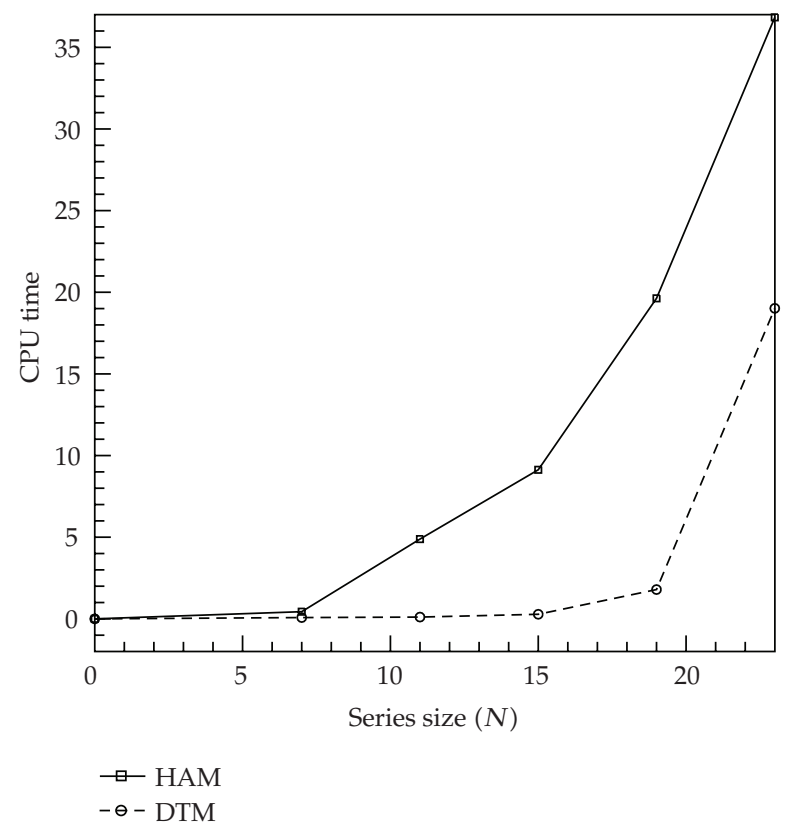

Figure 8: Comparison between CPU times of the DTM and the HAM (5th-order approximation) in computation of $f(\eta)$. 


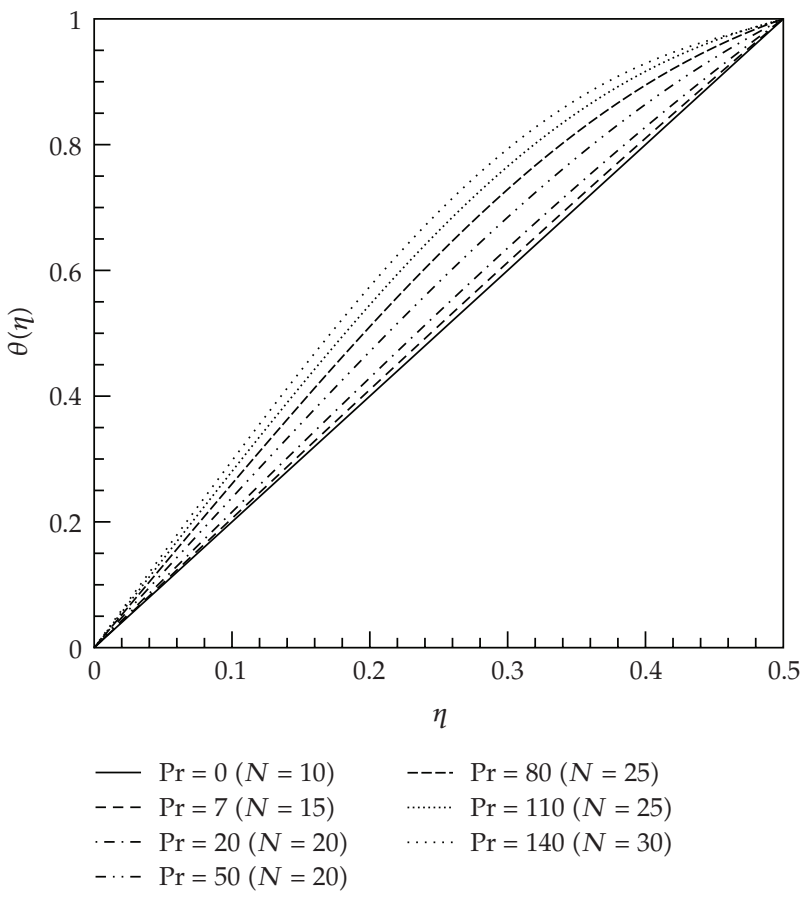

Figure 9: The normalized temperature profiles $(\theta(\eta))$ obtained by the DTM for different values of the Prandtl number, when $\delta=0.5$.

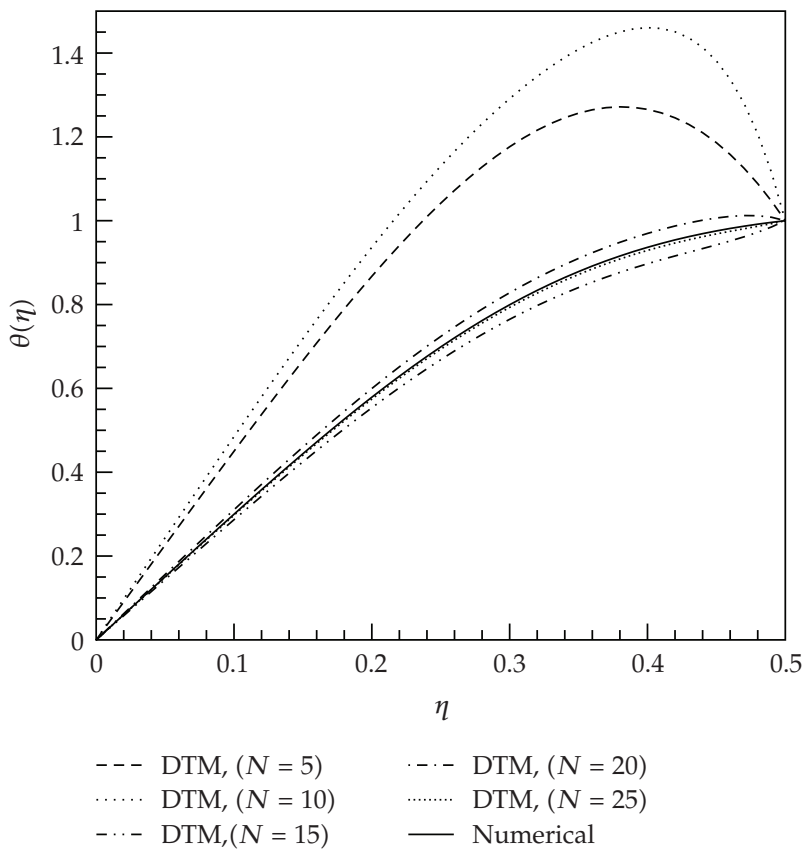

Figure 10: The normalized temperature profiles $(\theta(\eta))$ obtained by the DTM for different values of series size, when $\delta=0.5, \operatorname{Pr}=140$. 
Table 1: The analytic results of $f(\eta), g(\eta), k(\eta)$ and $s(\eta)$ at different values of series size compared with the numerical results, when $\delta=1$.

\begin{tabular}{|c|c|c|c|c|c|c|c|c|c|c|}
\hline & \multirow[t]{2}{*}{$\eta$} & \multicolumn{2}{|c|}{$N=5$} & \multicolumn{2}{|c|}{$N=10$} & \multicolumn{2}{|c|}{$N=15$} & \multicolumn{2}{|c|}{$N=20$} & \multirow[b]{2}{*}{ Numerica } \\
\hline & & DTM & HAM & DTM & HAM & DTM & HAM & DTM & HAM & \\
\hline \multirow{5}{*}{$f(\eta)$} & 0.2 & 0.012109 & 0.025333 & 0.012829 & 0.015790 & 0.012824 & 0.013583 & 0.012824 & 0.012675 & 0.012824 \\
\hline & 0.4 & 0.043688 & 0.093354 & 0.046554 & 0.057037 & 0.046536 & 0.049058 & 0.046535 & 0.045894 & 0.046535 \\
\hline & 0.6 & 0.088436 & 0.192237 & 0.094802 & 0.115414 & 0.094765 & 0.099494 & 0.094762 & 0.093341 & 0.094762 \\
\hline & 0.8 & 0.14108 & 0.310623 & 0.152095 & 0.18384 & 0.152036 & 0.159107 & 0.152032 & 0.149674 & 0.152032 \\
\hline & 1 & 0.197281 & 0.438095 & 0.213659 & 0.256624 & 0.213582 & 0.223019 & 0.213575 & 0.210237 & 0.213575 \\
\hline \multirow{5}{*}{$g(\eta)$} & 0.2 & 0.926037 & 0.8692 & 0.927126 & 0.906846 & 0.926989 & 0.913032 & 0.926994 & 0.916605 & 0.926994 \\
\hline & 0.4 & 0.860691 & 0.752533 & 0.863394 & 0.826667 & 0.863116 & 0.837423 & 0.863125 & 0.843679 & 0.863125 \\
\hline & 0.6 & 0.809872 & 0.6612 & 0.814989 & 0.767516 & 0.814561 & 0.780547 & 0.814575 & 0.788272 & 0.814575 \\
\hline & 0.8 & 0.777414 & 0.6032 & 0.785359 & 0.732751 & 0.784778 & 0.746104 & 0.784796 & 0.754295 & 0.784795 \\
\hline & 1 & 0.766044 & 0.583333 & 0.775603 & 0.721816 & 0.774925 & 0.734846 & 0.774945 & 0.743051 & 0.774944 \\
\hline \multirow{5}{*}{$k(\eta)$} & 0.2 & 0.160806 & 0.199372 & 0.158485 & 0.175442 & 0.158442 & 0.171012 & 0.158444 & 0.168154 & 0.158444 \\
\hline & 0.4 & 0.283364 & 0.355359 & 0.278667 & 0.30897 & 0.27858 & 0.301266 & 0.278584 & 0.296762 & 0.278584 \\
\hline & 0.6 & 0.369227 & 0.465866 & 0.362176 & 0.400584 & 0.362040 & 0.391304 & 0.362047 & 0.386586 & 0.362047 \\
\hline & 0.8 & 0.419793 & 0.5306 & 0.41076 & 0.452406 & 0.410574 & 0.443034 & 0.410583 & 0.438927 & 0.410583 \\
\hline & 1 & 0.436365 & 0.551389 & 0.426452 & 0.468504 & 0.426232 & 0.443034 & 0.426244 & 0.455797 & 0.426243 \\
\hline \multirow{5}{*}{$-s(\eta)$} & 0.2 & 0.046495 & -0.04397 & 0.044369 & 0.002434 & 0.044329 & 0.016543 & 0.044332 & 0.037467 & 0.044332 \\
\hline & 0.4 & 0.087213 & -0.07162 & 0.083042 & 0.009616 & 0.082966 & 0.032601 & 0.082971 & 0.070290 & 0.082971 \\
\hline & 0.6 & 0.118325 & -0.08646 & 0.112294 & 0.017710 & 0.112187 & 0.044910 & 0.112194 & 0.094853 & 0.112194 \\
\hline & 0.8 & 0.137666 & -0.09219 & 0.130157 & 0.023955 & 0.130029 & 0.052118 & 0.130038 & 0.109565 & 0.130038 \\
\hline & 1 & 0.144217 & -0.09305 & 0.136051 & 0.026361 & 0.135916 & 0.054308 & 0.135926 & 0.114315 & 0.135925 \\
\hline
\end{tabular}

\section{Nomenclature}

$f, g, k, s$ and $\theta$ : Nondimensional functions of $\eta$

$F(k): \quad$ Differential transforms of $f(\eta)$

$G(k): \quad$ Differential transforms of $g(\eta)$

$K(k): \quad$ Differential transforms of $k(\eta)$

$S(k): \quad$ Differential transforms of $s(\eta)$

$\Theta(k): \quad$ Differential transforms of $\theta(\eta)$

$\bar{g}: \quad$ Gravitational acceleration

$h$ : Thickness of fluid film

$W$ : $\quad$ Velocity of spraying

Pr: $\quad$ Prandtl number

$p_{0}: \quad$ Ambient pressure on the film surface

$T: \quad$ Temperature

$T_{0}$ : Temperature on the film surface

$T_{w}: \quad$ Temperature on the disk

$u$ : Velocity components in the $x$ direction

$v$ : $\quad$ Velocity components in the $y$ direction

$w$ : Velocity components in the $z$ direction. 


\title{
Greek symbols
}

\author{
$\Omega: \quad$ Angular velocity \\ $\alpha: \quad$ Thermal diffusivity of the fluid \\ $\beta$ : $\quad$ Angle between horizontal axis and disk \\ $\gamma_{i}(i=1, \ldots, 5)$ : Constants of the DTM \\ $\eta$ : $\quad$ Nondimensional variable \\ $\delta$ : $\quad$ Constant normalized thickness.
}

\section{Acknowledgment}

The authors express their gratitude to the anonymous referees for their constructive reviews of the manuscript and for helpful comments.

\section{References}

[1] A. H. Nayfeh, Introduction to Perturbation Techniques, John Wiley \& Sons, New York, NY, USA, 1979.

[2] R. H. Rand and D. Armbruster, Perturbation Methods, Bifurcation Theory and Computer Algebra, vol. 65 of Applied Mathematical Sciences, Springer, New York, NY, USA, 1987.

[3] J. K. Zhou, Differential Transformation and Its Applications for Electrical Circuits, Huazhong University Press, Wuhan, China, 1986.

[4] C. K. Chen and S. H. Ho, "Solving partial differential equations by two-dimensional differential transform method," Applied Mathematics and Computation, vol. 106, no. 2-3, pp. 171-179, 1999.

[5] F. Ayaz, "Solutions of the system of differential equations by differential transform method," Applied Mathematics and Computation, vol. 147, no. 2, pp. 547-567, 2004.

[6] I. H. Abdel-Halim Hassan, "Comparison differential transformation technique with Adomian decomposition method for linear and nonlinear initial value problems," Chaos, Solitons and Fractals, vol. 36, no. 1, pp. 53-65, 2008.

[7] A. S. V. Ravi Kanth and K. Aruna, "Solution of singular two-point boundary value problems using differential transformation method," Physics Letters. A, vol. 372, no. 26, pp. 4671-4673, 2008.

[8] A. Arikoglu and I. Ozkol, "Solution of differential-difference equations by using differential transform method," Applied Mathematics and Computation, vol. 181, no. 1, pp. 153-162, 2006.

[9] A. Arikoglu and I. Ozkol, "Solution of boundary value problems for integro-differential equations by using differential transform method," Applied Mathematics and Computation, vol. 168, no. 2, pp. 1145-1158, 2005.

[10] N. Bildik, A. Konuralp, F. O. Bek, and S. Küçükarslan, "Solution of different type of the partial differential equation by differential transform method and Adomian's decomposition method," Applied Mathematics and Computation, vol. 172, no. 1, pp. 551-567, 2006.

[11] M. M. Rashidi, S. A. Mohimanian pour, and S. Abbasbandy, "Analytic Approximate Solutions for Heat Transfer of a Micropolar Fluid through a Porous Medium with Radiation," Communications in Nonlinear Science and Numerical Simulations. In Press.

[12] M. M. Rashidi, "Differential transform method for solving two dimensional viscous flow," in Proceedings of the International Conference on Applied Physics and Mathematics, 2009.

[13] M. M. Rashidi, "Differential transform method for MHD boundary-layer equations: combination of the DTM and the Padé approximant," in Proceedings of the International Conference on Applied Physics and Mathematics, 2009.

[14] M. M. Rashidi and E. Erfani, "A novel analytical solution of the thermal boundary-layer over a flat plate with a convective surface boundary condition using DTM-Pade," in Proceedings of the International Conference on Applied Physics and Mathematics, 2009.

[15] E. M. Sparrow and J. L. Gregg, "A theory of rotating condensation," A Theory of Rotating Condensation, vol. 81, pp. 113-120, 1959.

[16] T. von Karman, "Uber laminare und turbulente reibung," Zeitschrift für Angewandte Mathematik und Mechanik, vol. 1, pp. 233-252, 1921.

[17] P. M. Beckett, P. C. Hudson, and G. Poots, "Laminar film condensation due to a rotating disk," Journal 
of Engineering Mathematics, vol. 7, no. 1, pp. 63-73, 1973.

[18] S. P. Chary and P. K. Sarma, "Condensation on a rotating disk with constant axial suction," Journal of Heat Transfer, vol. 98, no. 4, pp. 682-684, 1976.

[19] K. F. Jensen, E. O. Einset, and D. I. Fotiadis, "Flow phenomena in chemical vapor deposition of thin films," Annual Review of Fluid Mechanics, vol. 23, no. 1, pp. 197-232, 1991.

[20] C. Y. Wang, "Condensation film on an inclined rotating disk," Applied Mathematical Modelling, vol. 31, no. 8, pp. 1582-1593, 2007.

[21] M. M. Rashidi and S. Dinarvand, "Purely analytic approximate solutions for steady three-dimensional problem of condensation film on inclined rotating disk by homotopy analysis method," Nonlinear Analysis, vol. 10, no. 4, pp. 2346-2356, 2009.

[22] A. Arikoglu, G. Komurgoz, and I. Ozkol, "Effect of slip on the entropy generation from a single rotating disk," Journal of Fluids Engineering, Transactions of the ASME , vol. 130, no. 10, pp. 10120211012029, 2008.

[23] A. Arikoglu and I. Ozkol, "On the MHD and slip flow over a rotating disk with heat transfer," International Journal of Numerical Methods for Heat and Fluid Flow, vol. 16, no. 2, pp. 172-184, 2006. 


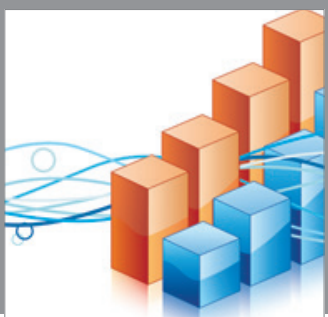

Advances in

Operations Research

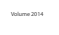

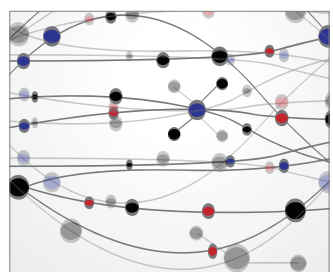

\section{The Scientific} World Journal
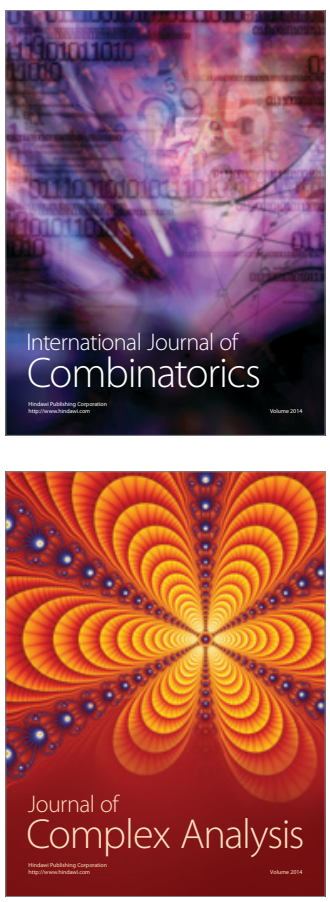

International Journal of

Mathematics and

Mathematical

Sciences
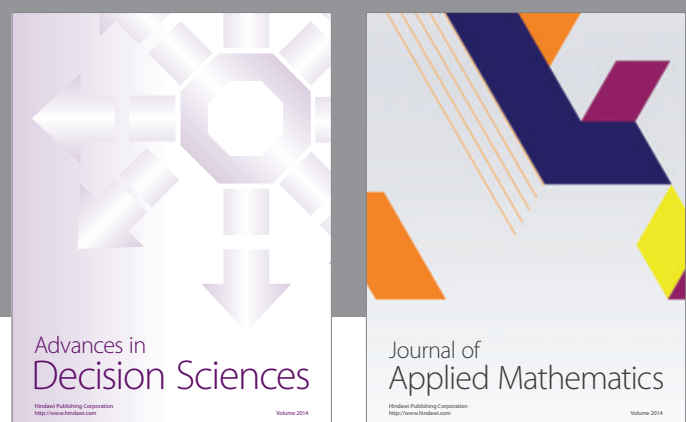

Journal of

Applied Mathematics
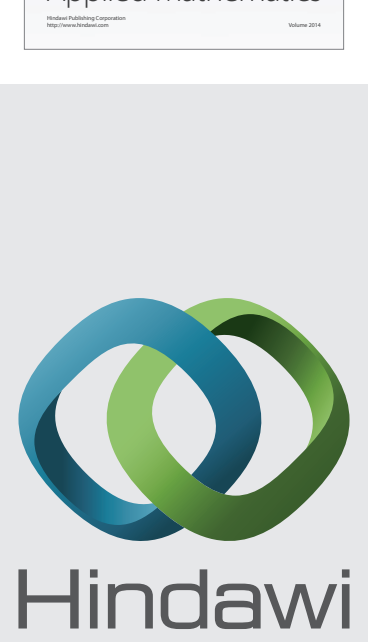

Submit your manuscripts at http://www.hindawi.com
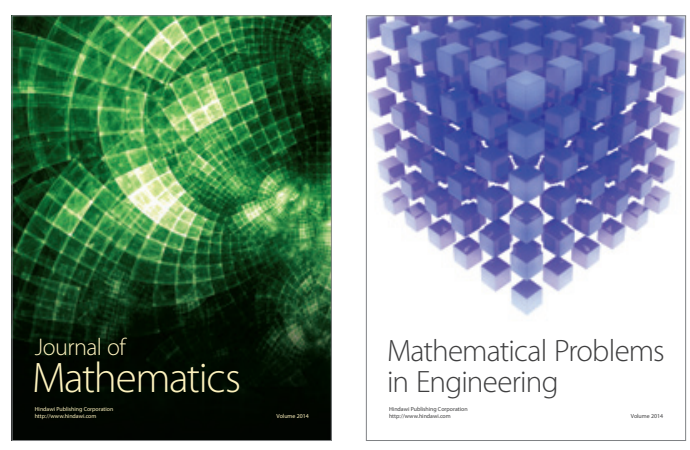

Mathematical Problems in Engineering
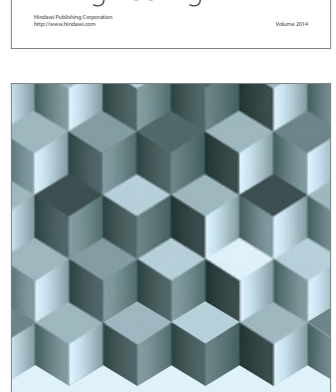

Journal of

Function Spaces
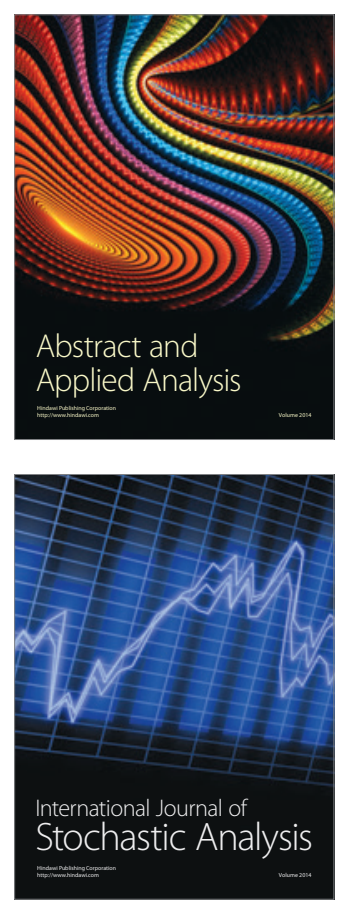

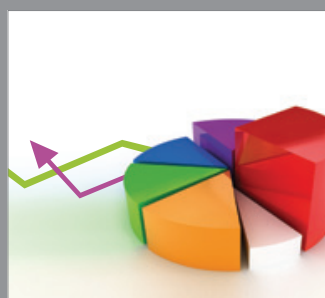

ournal of

Probability and Statistics

Promensencen
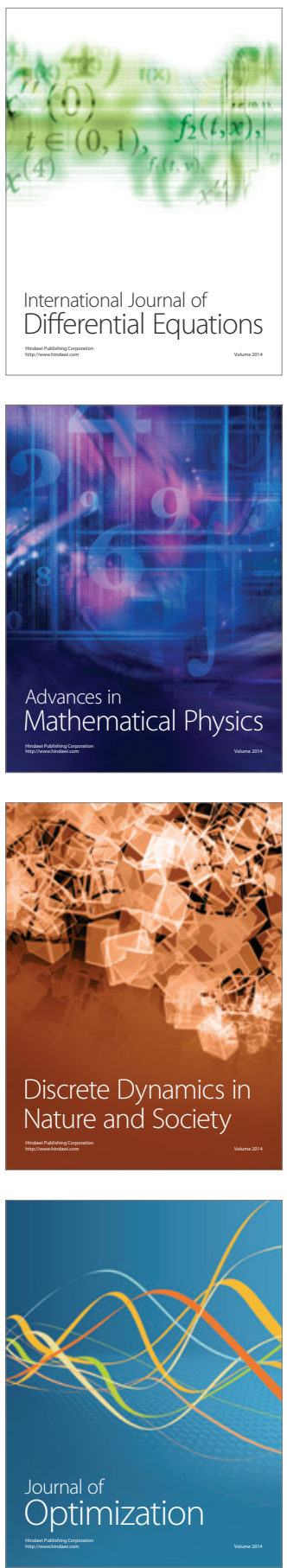